\title{
Effect of Acupuncture Combined with Tuina on Cervical Curvature and Serum Inflammatory Cytokine Levels in Patients with Cervical Spondylosis
}

\author{
YING LI' ${ }^{1}$ Y. Q. WU1, Y. Q. XIANG ${ }^{1}$ AND S. N. ZHANG* \\ Affiliated Hospital of Nanjing University of Chinese Medicine, Nanjing, Jiangsu 210029, ${ }^{2}$ School of rehabilitation medicine, Gannan \\ Medical University, Ganzhou, Jiangxi 341000, China
}

Li et al.: Acupuncture Combined with Tuina Effect in Cervical Spondylosis Patients

\begin{abstract}
To study the effects of acupuncture and tuina by stages on cervical curvature, serum interleukin-4, interleukin-8 and tumor necrosis factor-alpha in patients with cervical spondylosis. A total of 94 patients with cervical spondylosis admitted from June 2020 to June 2021 were divided into the control group and the study group through random number table with 47 cases in each group. Patients in the control group were treated with tuina and patients in the study group were treated with acupuncture and tuina by stages. One course of treatment was $21 \mathrm{~d}$ and both groups were treated for $\mathbf{2}$ courses. The clinical effects of the patients in the two groups after treatment were compared, the cervical spine curvature and visual analogue scale scores of patients in the two groups before and after treatment were compared and the serum levels of interleukin-4, interleukin-8 and tumor necrosis factor-alpha before and after treatment were compared. The total clinical effective rate of patients in the study group was $91.48 \%(43 / 47)$, which was higher than 68.09\% (32/47) of patients in the control group. After treatment, the $\mathbf{C}$ value of cervical spine physiological curvature of patients in the two groups was higher than that before treatment and the visual analog scale scores, serum interleukin-4, interleukin-8, tumor necrosis factor-alpha levels decreased. The improvement of patients in the study group was better than that in the control group (all $\mathbf{p}<\mathbf{0 . 0 5}$ ). Acupuncture and tuina to treat patients with cervical spondylosis by stages can better improve the curvature of the patient's cervical spines, reduce pain, inhibit inflammation in the body and improve the therapeutic effect.
\end{abstract}

Key words: Cervical spondylosis, acupuncture, tuina, cervical curvature, interleukin-4, interleukin-8, tumor necrosis factor-alpha

Cervical spondylosis is a kind of cervical syndrome, which is more common clinically. Cervical spondylosis is mainly caused by the long term strain of the cervical spine muscles, the loosening of the cervical ligaments, the instability of the vertebral body joints and other factors resulting in the stenosis of the vertebral body lumen, which then compresses the nerves and finally leads to a series of lesions ${ }^{[1,2]}$. Non-surgical therapy is an important treatment for radiculopathy. Western medicine mainly focuses on nourishing the nerves and eliminating the edema. Although the short-term effect is acceptable, it is easy to relapse after stopping the drug and the side effects are obvious ${ }^{[2]}$. In recent years, traditional Chinese medicine has been widely used in clinics, with characteristics such as syndrome differentiation and treatment and overall concepts, of which, acupuncture and tuina can exert effects on health care, health preservation and treatment. Tuina is a non- medicinal natural therapy; acupuncture is also a commonly used external treatment method for Chinese medicine, which can ventilate and activate the collaterals, strengthen blood perfusion, improve local blood circulation and effectively nourish the veins. It can effectively control the condition in the treatment of arthralgia and scapulohumeral periarthritis and has a certain degree of safety. However, whether the combined treatment of the two currently has a repeated effect and the clinical effect are controversial. This study aimed to investigate the effects of acupuncture combined with tuina on cervical curvature, Interleukin (IL)-4, IL-8 and Tumor Necrosis Factor-Alpha (TNF- $\alpha$ ) in patients with cervical spondylosis. The 94 patients with cervical spondylosis admitted to our hospital from June 2020 to June 2021 were selected. Meet the diagnostic criteria of cervical spondylosis ${ }^{[3]}$; pain and

*Address for correspondence

E-mail: zhangshinian@sina.com 
numbness were obvious and corresponded to cervical spinal nerve innervation and blood stasis, upper limb traction tension test and neck compression test were positive. Magnetic Resonance Imaging (MRI) showed local pathological anatomy. X-ray film showed bone spur formation, instability of vertebral segments and changes in cervical spine curvature; blood system and immune system were normal. Obvious pathological changes in major organs such as liver and kidney; breast-feeding or pregnancy; acute trauma or inflammation. According to the random number table method, they were divided into control group and study group with 47 cases in each group. In the control group, there were 21 females and 26 males; they were 35-68 y old, with an average of $47.20 \pm 6.32 \mathrm{y}$; the course of disease was 3-24 mo, with an average of 8.96 \pm 3.12 mo. In the study group, there were 24 females and 23 males; they were 34 to $67 \mathrm{y}$ old, with an average of $46.85 \pm 6.11$ $\mathrm{y}$; the course of disease was 4-24 mo, with an average of $9.25 \pm 3.65 \mathrm{mo}$. There was no statistical difference in basic data between patients in the two groups ( $p>0.05)$. Both groups of patients received conventional traction treatment measures and chose a seated jaw-occipital belt. In the initial stage, the traction force was about $1 / 10^{\text {th }}$ of the body weight and then it could be gradually increased, each time about 25 min of traction. Patients in the control group received tuina therapy. The physiotherapist should stand behind the patient and then treat the scapula, neck and other positions of the patient by using holding and dialing methods. In this process, the physiotherapist used the extension and rotation method. The patient adopted a sitting position, the physiotherapist placed his hands behind the pillow and under the jaw, the head was kept in a neutral state, the neck was slightly tilted forward and then pulled upward for $2 \mathrm{~min}$ and then tilted and slowly rotated left and right. At this stage, the physiotherapist communicated with the patient at any time, adjusted the intensity appropriately and rested for $1 \mathrm{~d}$ after $6 \mathrm{~d}$ of treatment. One course of treatment was $21 \mathrm{~d}$ and there were 2 consecutive courses of treatment. Patients in the study group were combined with acupuncture and tuina on the basis of conventional treatment and acupuncture treatment was performed first. Take the patients' Fengchi acupoint, neck Jiaji acupoint, Dazhui acupoint and Naokong acupoint and apply 1.2 inch needles. After routine disinfection to the patient, oblique puncture Fengchi acupoint 0.5-0.8 inch, straight puncture Jiaji acupoint 0.5-0.8 inch, oblique puncture Dazhui acupoint $0.8-1$ inch and flat puncture Naokong acupoint 0.3-0.5 inch. The method of replenishing and relieving was adopted and then the patient's qi was taken as the degree. After the needle was inserted, the moxa stick at the needle handle needed to be ignited, 15 $\mathrm{min} /$ time close to the acupuncture point and the patient should be communicated in a timely manner whether the temperature was appropriate. The treatment was performed once a day for 21 consecutive $\mathrm{d}$. After the acupuncture period over, it is necessary to continue to enter the tuina period. The method of tuina was the same as that in the control group. Patients rested for $1 \mathrm{~d}$ after $6 \mathrm{~d}$ of treatment. One course of treatment was $21 \mathrm{~d}$ and there were 2 consecutive courses of treatment and then all patients continued treatment for 1 course of treatment, followed by the evaluation of various indicators. The treatment effects of patients in the two groups after treatment (after two courses of treatment) were compared. The criteria for determining the curative effect were as follows ${ }^{[4]}$. Those whose clinical symptoms disappeared and work and life returned to normal and who did not relapse during the follow-up period were regarded as cured, those whose clinical symptoms were significantly reduced without affecting work and life and who occasionally relapsed during the follow-up period and were relieved after treatment were regarded as improved; those whose clinical symptoms were unchanged or slightly alleviated and work and life were still affected were regarded as ineffective. Total effective rate $=$ cure rate + improvement rate. The changes in symptom scores of patients in the two groups before and after treatment were observed and the two secondary symptoms of each were divided into 4 levels. The main symptoms were $0,2,4$ and 6 and the secondary symptoms were $0,1,2$ and 3 . The traditional Chinese medicine symptom score was the sum of two scores and the higher the score shows the better effect. Before and after the treatment, under the same conditions, the patient's cervical X-ray lateral view was taken to assess the curvature of the cervical spine. Draw a straight line from the upper edge of the posterior odontoid process of the axis to the lower edge of the seventh cervical vertebra (C7 vertebral body), which was line A. Draw a line along the posterior edge of each cervical vertebral body as line $\mathrm{B}$. The vertical cross line at the widest point between lines $\mathrm{A}$ and $\mathrm{B}$ was $\mathrm{C}$ line and the normal range of $\mathrm{C}$ line (C value) was $(12.00 \pm 5.00) \mathrm{mm}^{[5]}$. The Visual Analog Scale (VAS) ${ }^{[6]}$ was used to evaluate the pain value: For $0-10$ points, the lower the score, the lower the pain. The $5 \mathrm{ml}$ of fasting venous blood from patients in the two groups before and after treatment was collected, the blood samples were centrifuged and the serum (centrifuged at $3500 \mathrm{r} / \mathrm{min}$ for $10 \mathrm{~min}$ ) was 
obtained. The detection method of serum levels of IL-4, IL-8 and TNF- $\alpha$ was Enzyme-Linked Immunosorbent Assay (ELISA). During the detection process, attention should be paid to strictly follow the detection instructions of the kit. The Statistical Package for Social Sciences (SPSS) 20.0 statistical software was used to process the data and the measurement data was represented by $(\mathrm{x} \pm \mathrm{s})$ using $\mathrm{t}$ test; the count data was represented by [no. (\%)] using $\chi^{2}$ test; $p<0.05$ indicated that the difference was statistically significant. After treatment, the total clinical effective rate of patients in the study group was $91.48 \%$ (43/47), which was significantly higher than $68.09 \%(32 / 47)$ in the control group $(\mathrm{p}<0.01)$, as shown in Table 1 . Before treatment, patients in the two groups had no difference in dizziness, nausea or vomiting, headache, shoulder and neck pain, neck rotation test, life and work scores $(p>0.05)$; the above indicators of patients in the two groups were higher after treatment than those before treatment and the indicators in the study group was higher than those in the control group $(p<0.05)$, as shown in Table 2. The $\mathrm{C}$ value of cervical spine physiological curvature of patients in the two groups was higher and the pain scores were lower than those before treatment. The improvement in the study group was better than that in the control group. The differences were statistically significant (all $\mathrm{p}<0.05$ ), as shown in Table 3. After treatment, the levels of serum inflammatory cytokines of patients in the two groups were lower than those before treatment. The levels of serum inflammatory cytokines of patients in the study group were lower than those in the control group. The differences were statistically significant (all $\mathrm{p}<0.05$ ), as shown in Table 4. Cervical osteoarthritis, cervical nerve root syndrome, proliferative cervical spondylitis and cervical disc herniation are all cervical spondylosis ${ }^{[7]}$. Cervical spondylosis is a disease based on degenerative pathological changes, which is also referred to as the cervical spine syndrome clinically, and its condition is protracted and unhealed, which has a serious impact on the daily life and health of the patients ${ }^{[8]}$. The onset of cervical spondylosis is related to the patient's bone hyperplasia, ligament thickening, long-term cervical strain, etc. Long-term compression of the cervical spine and spinal cord can cause poor blood flow in the vertebral artery and compression of the nerve roots, which in turn causes the patients to experience various clinical symptoms and manifestations ${ }^{[9]}$. From the perspective of traditional Chinese medicine, cervical spondylosis belongs to the category of "paralysis". The disease is caused by trauma and strain, loss of qi and blood, Feng Xie invasion, etc. After Shi Xie invasion and the obstruction of the meridian, the patients will suffer from insufficient qi and blood and blocking of the meridians. The main treatment principles should be activating blood and dredging collaterals, reducing swelling and pain and dispelling wind and cold. Only then the curative effect can be achieved. The scapula and neck of the patients can be treated by the holding and dialing methods in tuina, relieving the tendons and collaterals, relieving sweating, sedation and reliving pain, normalizing the muscles and restoring and smoothing the joints ${ }^{[10]}$; acupuncture at Fengchi acupoint can regulate qi and blood and unblock the veins; acupuncture at Jiaji acupoint on the neck can improve blood circulation, relax nerves and improve blood circulation; acupuncture at Dazhui can promote qi and blood circulation and relieve pain; acupuncture at Naokong acupoint can relieve wind-heat, soothes the skin and promote sweating. Studies have shown that the combined application of tuina and acupuncture can alleviate the pain symptoms of patients, effectively improve the therapeutic effects of patients, relieve the nerve compression of patients and help patients improve the symptoms of cervical spondylosis and improve the curvature of the cervical spine ${ }^{[11]}$. The results of this study showed that the clinical effects and $\mathrm{C}$ value of cervical spine physiological curvature of patients in the study group were higher than those in the control group after treatment and the VAS scores of patients in the study group were lower than those in the control group, suggesting that combined application of tuina and acupuncture can relieve the clinical symptoms of patients with cervical spondylosis, relieve pain, improve the cervical spine physiological curvature and restore cervical spine function. Serum IL-4, IL- 8 and TNF- $\alpha$ are the main cytokines of inflammatory response, which can participate in the transmission of sensory information in time and are highly sensitive to pain and inflammation. Studies have shown that serum IL-4, IL-8 and TNF- $\alpha$ will increase rapidly with the development of cervical spondylosis. Once patients with cervical spondylopathy suffer from the disease for a long time, the blood vessels and nerves will be squeezed, resulting in local exudation of inflammatory cytokines and chronic head and neck injuries ${ }^{[12]}$. The combined application of acupuncture and tuina can relax the patient's body veins and can also promote qi and blood circulation, thereby strengthening blood perfusion, nourishing muscles and veins, improving blood circulation and regulating the body's inflammatory response ${ }^{[13]}$. In this study, the levels of inflammatory 
cytokines of patients in the study group after treatment were lower than those in the control group, suggesting that the combined application of tuina and acupuncture can inhibit the inflammatory response of patients with cervical spondylosis and promote the recovery from the disease. In conclusion, the combined application of tuina and acupuncture in the treatment of patients with cervical spondylosis can reduce the patients' pain, improve the physiological curvature of the cervical spine, inhibit inflammation and enhance the therapeutic effects, which is worthy of application and promotion.

TABLE 1: CLINICAL EFFECTS BETWEEN PATIENTS IN THE TWO GROUPS

\begin{tabular}{lccccc}
\hline Group & $\mathrm{n}$ & Cured & Improved & Ineffective & Effective rate \\
\hline Study group & 47 & 29 & 15 & 3 & $43(91.48)$ \\
Control group & 47 & 19 & 13 & 15 & $32(68.09)$ \\
F & & & 14.673 & & \\
$\mathrm{p}$ & & & $<0.01$ & \\
\hline
\end{tabular}

TABLE 2: SYMPTOM SCORES BETWEEN PATIENTS IN THE TWO GROUPS

\begin{tabular}{lccccc}
\hline Group & & Study group & Control group & F & P \\
\hline Dizziness & Before treatment & $4.57 \pm 0.52$ & $4.59 \pm 0.49$ & 0.594 & 0.55 \\
& After treatment & $9.10 \pm 0.92$ & $13.27 \pm 1.2$ & 56.7 & 0 \\
Nausea or vomiting & Before treatment & $4.28 \pm 0.59$ & $4.33 \pm 0.62$ & 1.239 & 0.22 \\
& After treatment & $5.28 \pm 0.57$ & $5.72 \pm 0.48$ & 12.53 & 0 \\
\hline
\end{tabular}

TABLE 3: CERVICAL SPINE PHYSIOLOGICAL CURVATURE C VALUE AND PAIN SCORE BETWEEN PATIENTS IN THE TWO GROUPS

\begin{tabular}{lccccc}
\hline \multirow{2}{*}{ Group } & & \multicolumn{2}{c}{$\begin{array}{c}\text { Cervical spine physiological curvature C } \\
\text { value }(\mathrm{mm})\end{array}$} & \multicolumn{2}{c}{ Pain score (point) } \\
\cline { 3 - 6 } & $\mathrm{n}$ & Before treatment & After treatment & Before treatment & After treatment \\
\hline Study group & 47 & $3.52 \pm 2.52$ & $11.34 \pm 3.8$ & $7.31 \pm 2.52$ & $1.89 \pm 0.55$ \\
Control group & 47 & $3.35 \pm 3.68$ & $5.64 \pm 3.84$ & $7.12 \pm 2.68$ & $2.25 \pm 0.75$ \\
$\mathrm{t}$ & & 0.238 & 6.58 & 0.322 & 2.417 \\
\hline
\end{tabular}

TABLE 4: INFLAMMATORY CYTOKINES BETWEEN PATIENTS IN THE TWO GROUPS

\begin{tabular}{|c|c|c|c|c|c|c|c|}
\hline \multirow[b]{2}{*}{ Group } & \multirow[b]{2}{*}{$\mathrm{n}$} & \multicolumn{2}{|c|}{ IL-4 (ng/l) } & \multicolumn{2}{|c|}{ IL-8 (ng/l) } & \multicolumn{2}{|c|}{ TNF-a (nmol/l) } \\
\hline & & $\begin{array}{c}\text { Before } \\
\text { treatment }\end{array}$ & $\begin{array}{c}\text { After } \\
\text { treatment }\end{array}$ & $\begin{array}{c}\text { Before } \\
\text { treatment }\end{array}$ & $\begin{array}{c}\text { After } \\
\text { treatment }\end{array}$ & $\begin{array}{c}\text { Before } \\
\text { treatment }\end{array}$ & $\begin{array}{c}\text { After } \\
\text { treatment }\end{array}$ \\
\hline Study group & 47 & $37.94 \pm 6.03$ & $13.84 \pm 7.05$ & $0.94 \pm 0.35$ & $0.23 \pm 0.04$ & $9.64 \pm 4.84$ & $3.73 \pm 3.08$ \\
\hline Control group & 47 & $36.73 \pm 5.93$ & $21.73 \pm 5.96$ & $0.85 \pm 0.24$ & $0.43 \pm 0.06$ & $9.84 \pm 3.94$ & $5.62 \pm 2.05$ \\
\hline $\mathrm{t}$ & & 0.893 & 5.337 & 1.324 & 17.321 & 0.2 & 3.19 \\
\hline $\mathrm{p}$ & & $>0.05$ & $<0.05$ & $>0.05$ & $<0.05$ & $>0.05$ & $<0.05$ \\
\hline
\end{tabular}

\section{Acknowledgements:}

This work was supported by the scientific research project of traditional Chinese medicine of Jiangxi Provincial Health and Family Planning Commission (No. 2018A103).

\section{Conflicts of interest:}

The authors report no conflicts of interest.

\section{REFERENCES}

1. Nouri A, Tetreault L, Singh A, Karadimas SK, Fehlings MG. Degenerative cervical myelopathy: Epidemiology, genetics, and pathogenesis. Spine 2015;40(12):E675-93.

2. Nouri A, Martin AR, Tetreault L, Nater A, Kato S, Nakashima $\mathrm{H}$, et al. MRI analysis of the combined prospectively collected AOSpine North America and International Data: The prevalence and spectrum of pathologies in a global cohort of patients with degenerative cervical myelopathy. Spine 2017;42(14):1058-67.

3. Luo J, Cao K, Huang S, Li L, Yu T, Cao C, et al. Comparison of anterior approach versus posterior approach for the treatment of multilevel cervical spondylotic myelopathy. Eur Spine J 2015;24(8):1621-30. 
4. Angevine PD, Arons RR, McCormick PC. National and regional rates and variation of cervical discectomy with and without anterior fusion, 1990-1999 Spine 2003;28(9):931-9.

5. Korinth MC. Treatment of cervical degenerative disc diseasecurrent status and trends. Zentralbl Neurochir 2008;69(03):11324.

6. Brkaric M, Baker KC, Israel R, Harding T, Montgomery DM, Herkowitz HN. Early failure of bioabsorbable anterior cervical fusion plates: Case report and failure analysis. Clin Spine Surg 2007;20(3):248-54.

7. Bartels RH, Donk R. Fusion around cervical disc prosthesis: case report. Neurosurgery 2005;57(1):E194.

8. Cheung ZB, Gidumal S, White S, Shin J, Phan K, Osman N, et al. Comparison of anterior cervical discectomy and fusion with a stand-alone interbody cage versus a conventional cage-plate technique: A systematic review and meta-analysis. Global Spine J 2019;9(4):446-55.

9. Barbagallo GM, Romano D, Certo F, Milone P, Albanese V. Zero-P: a new zero-profile cage-plate device for single and multilevel ACDF. A single institution series with four years maximum follow-up and review of the literature on zeroprofile devices. Eur Spine J 2013;22(6):868-78.

10. Song KJ, Taghavi CE, Lee KB, Song JH, Eun JP. The efficacy of plate constructs augmentation versus cage alone in anterior cervical fusion. Spine 2009;34(26):2886-92.
11. Matz PG, Pritchard PR, Hadley MN. Anterior cervical approach for the treatment of cervical myelopathy. Neurosurgery 2007;60(1):S1-64.

12. Tasiou A, Giannis T, Brotis AG, Siasios I, Georgiadis I, Gatos $\mathrm{H}$, et al. Anterior cervical spine surgery-associated complications in a retrospective case-control study. J Spine Surg 2017;3(3):444-59.

13. Chen Y, Chen H, Wu X, Wang X, Lin W, Yuan W. Comparative analysis of clinical outcomes between zero-profile implant and cages with plate fixation in treating multilevel cervical spondilotic myelopathy: A three-year follow-up. Clin Neurol Neurosurg 2016;144:72-6.

This is an open access article distributed under the terms of the Creative Commons Attribution-NonCommercial-ShareAlike 3.0 License, which allows others to remix, tweak, and build upon the work non-commercially, as long as the author is credited and the new creations are licensed under the identical terms

This article was originally published in a special issue, "Novel Therapeutic Approaches in Biomedicine and Pharmaceutical Sciences" Indian J Pharm Sci 2021:83(6) Spl Issue "205-209" 